\title{
Nuclear magnetic transitions in the relativistic energy density functional ap- proach
}

\author{
Nils Paar $^{1, *}$, Goran Kružić ${ }^{2}$, and Tomohiro Oishi ${ }^{1}$ \\ ${ }^{1}$ Department of Physics, Faculty of Science, University of Zagreb, Bijenička c. 32, HR-10000, Zagreb, Croatia \\ ${ }^{2}$ Research department, Ericsson - Nikola Tesla, Krapinska 45, HR - 10000, Zagreb, Croatia
}

\begin{abstract}
Recently a novel theory framework has been established for description of magnetic dipole (M1) transitions in finite nuclei, based on relativistic nuclear energy density functional with point coupling interactions. The properties of M1 transitions have been studied, including the sum rules, spin, orbital, isoscalar and isovector M1 transition strengths in magic and open shell nuclei. It is shown that pairing correlations and spinorbit interaction plays an important role in the description of M1 transition strength distributions. The analysis of the evolution of M1 transition properties in the isotope chain ${ }^{100-140} \mathrm{Sn}$ shows the interplay between single and double-peak structures, determined by the evolution of single-particle states, their occupations governed by the pairing correlations, and two-quasiparticle transitions involved. Comparison of the calculated B(M1) transition strength with recent data from inelastic proton scattering on ${ }^{112-124} \mathrm{Sn}$, shows that quenching of the $g$ factors $g_{\text {eff }} / g_{\text {free }}=0.80-0.93$ is required to reproduce the experimental data. Further experimental investigations are needed to determine accurately the quenching factor.
\end{abstract}

\section{Introduction}

Magnetic dipole (M1) transitions represent fundamental excitation phenomena in finite nuclei. Because of their relevance for various nuclear properties and applications, M1 transitions have been extensively studied both theoretically and experimentally [1-6]. The isovector spin-flip M1 response is relevant for the understanding of singleparticle properties in nuclei, spin-orbit interaction, and shell closures from stable nuclei toward limits of stability [7-9]. It is also relevant for applications related to the design of nuclear reactors [10]. The M1 transitions are also important for resolving the problem of quenching of the spin-isospin response in nuclei that is necessary for reliable description of double beta decay matrix elements [11]. The M1 spin-flip excitations can also be used as a probe for inelastic neutrino-nucleus cross sections [12], and they are relevant in modelling the r-process nucleosynthesis $[6,13]$. In deformed nuclei, M1 scissors mode has been extensively studied, where the orbital part of M1 operator plays a dominant role, with protons and neutrons oscillating with opposite phase around the core $[2,5,14,15]$. Experimental studies of M1 transitions are rather challenging, due to simultaneous presence of electric dipole (E1) and electric quadrupole (E2) transitions, which dominate over the M1 response [2, 16-19].

Various theoretical approaches, mainly in the nonrelativistic framework, have been employed in studies of M1 transitions - for more details see Refs. [4, 9, 14, 20] and references therein. While Skyrme functionals successfully reproduce electric excitations, there are difficulties to

*e-mail: npaar@phy.hr describe magnetic transitions, and further improvements in the the spin channel are required [20]. A study of M1 strength functions based on D1M Gogny force showed that the available experimental data could be reproduced if the calculated strength is shifted globally by about $2 \mathrm{MeV}$ and increased by an empirical factor of 2 [6]. Clearly, further theoretical studies of M1 transitions are required to resolve open questions on their properties. To respond to some of the open challenges, recently a novel theory framework has been established for studies of M1 excitations, based on the relativistic nuclear energy density functional $[21,22]$. This framework has been employed in several studies, including the analysis of basic M1 properties, their isotopic dependence and relationship with spinorbit interaction, the role of pairing properties, constraining the quenching of the $g$ factors for the free nucleons, and others. One of the important advantages to employ the relativistic framework is the natural description of the spin-orbit splitting, which plays an essential role in the M1 transitions. Here we give only a brief overview of recent studies, for more details see Refs.[21-24].

\section{Magnetic dipole transitions in the relativistic quasiparticle random phase approximation}

The relativistic quasiparticle random phase approximation (RQRPA) for description of M1 transition strength, based on relativistic point coupling interactions, was introduced in Ref.[21]. The nuclear ground state properties are described using the relativistic Hartree-Bogoliubov (RHB) 
model with DD-PC1 interaction, that provides singleparticle states and wave functions obtained in the canonical basis for the implementation in the RQRPA [21]. In addition to the point coupling interaction DD-PC1 used in the RHB model [21], for the description of unnatural parity excitations of the M1 type $\left(J^{\pi}=1^{+}\right)$, the RQRPA residual interaction is supplemented with the relativistic isovector-pseudovector contact interaction,

$$
\mathcal{L}_{I V-P V}=-\frac{1}{2} \alpha_{I V-P V}\left[\bar{\Psi}_{N} \gamma^{5} \gamma^{\mu} \vec{\tau} \Psi_{N}\right] \cdot\left[\bar{\Psi}_{N} \gamma^{5} \gamma_{\mu} \vec{\tau} \Psi_{N}\right]
$$

Since this term does not contribute to the ground state due to parity arguments, its strength parameter $\alpha_{I V-P V}$ is constrained by the experimental data on M1 excitation energies of ${ }^{48} \mathrm{Ca}$ and ${ }^{208} \mathrm{~Pb}$ [21]. By solving the RQRPA equations in the matrix formulation, the M1 transition strengths are obtained and further analyzed. The M1 sum rule for core-plus-two-nucleon systems [25] has been used to validate the model calculations. The spin, orbital, isoscalar and isovector M1 transition strengths have been studied in detail in magic nuclei ${ }^{48} \mathrm{Ca}$ and ${ }^{208} \mathrm{~Pb}$, and open shell nuclei ${ }^{42} \mathrm{Ca}$ and ${ }^{50} \mathrm{Ti}$ [21]. In these systems, the isovector spin-flip M1 transition is dominant, mainly between one or two spin-orbit partner states. As an illustrative example, in Fig. 1 the M1 transition strength distributions are shown for ${ }^{208} \mathrm{~Pb}$, including the full response $\mathrm{R}(\mathrm{M} 1)$, spin response $\mathrm{R}_{\mathrm{M} 1}^{\sigma}(\mathrm{E})$, and orbital response $\mathrm{R}_{\mathrm{M} 1}^{\ell}(\mathrm{E})$ functions. For comparison, the unperturbed response without the RQRPA residual interaction (Hartree response) is also shown. The spin M1 response dominates in the transition strength, and the residual RQRPA interaction clearly separates the M1 response from the unperturbed one. Two main peaks are identified in the spectra, the low one at $6.11 \mathrm{MeV}$, with the major contribution from the transitions between spin-orbit partner states for neutrons, $\left(v 1 i_{13 / 2}^{-1} \rightarrow v 1 i_{11 / 2}\right)$ and protons, $\left(\pi 1 h_{11 / 2}^{-1} \rightarrow \pi 1 h_{9 / 2}\right)$, that interfere destructively. The second state at $7.51 \mathrm{MeV}$ is composed of coherent transitions $\left(v 1 i_{13 / 2}^{-1} \rightarrow v 1 i_{11 / 2}\right)$ and $\left(\pi 1 h_{11 / 2}^{-1} \rightarrow \pi 1 h_{9 / 2}\right)$ (for more details see Ref.[21]).

In further studies of open shell nuclei, it is shown that pairing correlations have a significant impact on the centroid energy and major peak position of the M1 mode $[21,22]$. From the analysis of M1 mode in the Ca isotope chain, the role of the isovector-pseudovector (IV-PV) residual interaction has been discussed [22]. It has been shown that the experimental data on M1 mode could improve and optimize the theoretical aspects to describe the residual interactions in the RQRPA. In Ref. [24] a relation between the M1 excitation of nuclei and the pairing modes used in model calculations has been explored. For nucleons, two modes of the Cooper-pair coupling exist, namely of $S_{12}=0$ with $L_{12}=0$ (spin-singlet s-wave) and $S_{12}=1$ with $L_{12}=1$ (spin-triplet $\mathrm{p}$-wave). It has been shown that the model calculation of M1 transitions, combined with accurate experimental data, allow to discern the role of the spin singlet and spin triplet pairing correlations [24].

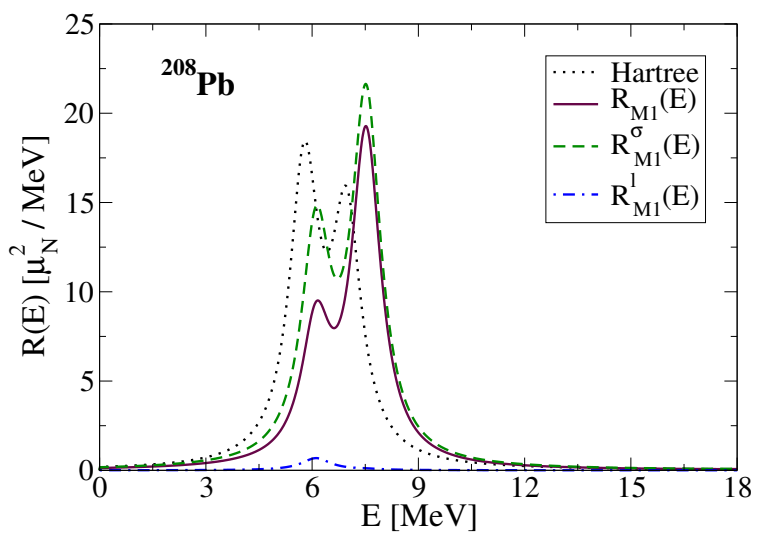

Figure 1. The $\mathrm{M} 1$ transition strength distributions for ${ }^{208} \mathrm{~Pb}$, including full response $\mathrm{R}_{\mathrm{M} 1}(\mathrm{E})$, spin response $\mathrm{R}_{\mathrm{M} 1}^{\sigma}(\mathrm{E})$, orbital response $\mathrm{R}_{\mathrm{M} 1}^{\ell}(\mathrm{E})$, and unperturbed (Hartree) response functions.

\section{Isotopic dependence of the M1 transition strength in ${ }^{100-140} \mathrm{Sn}$ and quenching of the g-factors}

Recent experimental study using inelastic proton scattering on Sn isotopes provided novel data on E1 and M1 strength distributions along the even-even ${ }^{112-124}$ Sn nuclei [26]. Since the resulting photoabsorption cross sections derived from the E1 and M1 strength distributions showed significant differences when compared to those from previous $(\gamma, x n)$ experiments $[27,28]$, it is interesting to explore how the new experimental data compare to the model calculations based on the relativistic nuclear energy density functional. Resolving the properties of M1 modes of excitation using new experimental data is also essential for our understanding of the role of M1 transitions in modeling radiative neutron capture cross sections of relevance for nucleosynthesis. Therefore, in Ref. [23] the M1 transitions have been investigated in even-even ${ }^{100-140} \mathrm{Sn}$ isotopes using the RQRPA. It has been shown that the M1 transition strength distribution is characterized by an interplay between single and double-peak structures, that can be understood from the evolution of single-particle states, their occupations governed by the pairing correlations, and twoquasiparticle transitions involved [23].

Figure 2 shows the evolution of transition strengths for two dominant M1 peaks along Sn isotope chain. Starting from ${ }^{102} \mathrm{Sn}$, the strength of the low- (high-) energy peak is increasing (decreasing), until its maximal (minimal) value is obtained at ${ }^{116} \mathrm{Sn}$. Further, the strength continues decreasing (increasing) until doubly magic ${ }^{132} \mathrm{Sn}$, where the trend reverses again. From the analysis of the relevant transitions, one can understand the evolution of the strengths of two main peaks. The low-energy state is characterizd by destructive interference between dominating nearly constant proton contributions and smaller neutron contributions that display variation along the isotope chain. The evolution of high-energy state is a result of dominating neutron contributions followed by the same behavior of smaller proton contributions. Different structures of the low- and high-energy M1 state govern different 


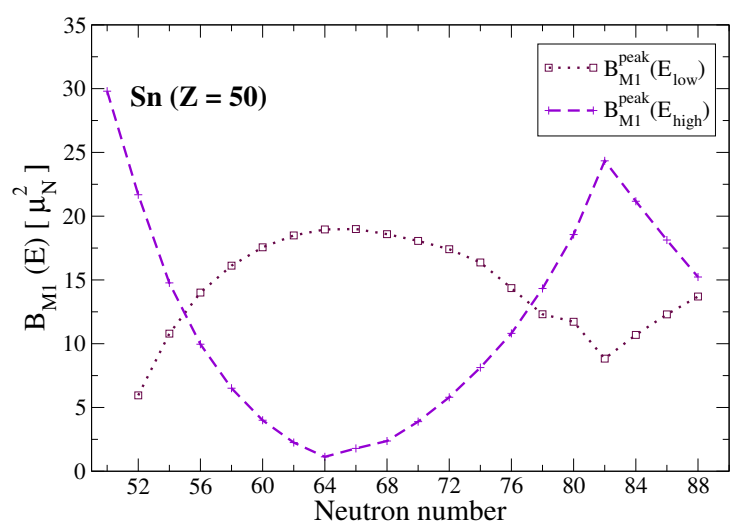

Figure 2. The M1 transition strengths for the two main peaks in even-even ${ }^{100-140} \mathrm{Sn}$ isotopes, where $E_{\text {low }}$ and $E_{\text {high }}$ denote energies of the low- and high-energy states.

evolution of their strengths along the Sn isotope chain. The M1 excitations are composed from transitions between the spin-orbit (SO) partner states. Therefore, it is interesting to explore the relationship between the SO energy splittings and $\mathrm{R}(\mathrm{Q}) \mathrm{RPA}$ excitation energies along the $\mathrm{Sn}$ isotope chain, as shown in Fig. 3. The M1 excitations induced by spin-flip transitions hold orbital quantum numbers unchanged. As shown in Fig. 3, the lower M1 state is very close to the proton SO splitting energy $\left(1 g_{9 / 2}\right)-\left(1 g_{7 / 2}\right)$, and the residual $\mathrm{R}(\mathrm{Q}) \mathrm{RPA}$ interaction only slightly reduces the M1 excitation energies with respect to the relevant SO splittings. However, in the case of second M1 state, the respective transitions are dominated by $v\left(1 g_{9 / 2}-1 g_{7 / 2}\right)$ configuration until ${ }^{116} \mathrm{Sn}$ and $v\left(1 h_{11 / 2}-1 h_{9 / 2}\right)$ configuration in heavier $\mathrm{Sn}$ isotopes. We note that their R(Q)RPAexcitation energies are different than the SO splitting energies of neutrons, thus showing the relevance of the IV-PV and pairing residual interactions in the RQRPA.

In the following the overall calculated B(M1) strength is compared to the recent experimental data from inelastic proton scattering [26]. The RQRPA summation of the B(M1) strength amounts 22.81, 22.61, 22.56, 22.76, 23.34, and $25.55 \mu_{N}^{2}$ for even-even isotopes ${ }^{112-124} \mathrm{Sn}$, respectively. The corresponding experimental values amount 14.7(1.4), 19.6 (1.9), 15.6 (1.3), 18.4 (2.4), 15.4(1.4), and 19.1(1.7) $\mu_{N}^{2}$, respectively. From the comparison of the RQRPA results with the experimental data, the effective $g$ factor can be obtained, that is needed to reproduce the experimental $\mathrm{B}(\mathrm{M} 1)$ transition strength. In this way one can determine the quenching factor as the ratio of the effective $g$ factor including quenching $\left(g_{\text {eff }}\right)$ with respect to the one for free nucleons $\left(g_{\text {free }}\right)$. With the assumption that the general quenching applies to all gyromagnetic factors in the M1 transition operator, the quenching factor is obtained, $g_{\text {eff }} / g_{\text {free }}=0.80-0.93$, that is higher value than previously reported, $g_{\text {eff }} / g_{\text {free }} \approx 0.6-$ 0.75 [2, 29-31]. Therefore, smaller quenching of the $g_{\text {free }}$ factor is needed to reproduce recent inelastic proton scattering data on M1 transitions. Since part of the B(M1) strength above the neutron threshold may be missing in the experiment, the present results show that the actual quenching of the $g$ factors in nuclei may be rather small in comparison to the ones for the free nucleons. Additional experimental studies are required to support this conclusion.

\section{Conclusion}

Recently established theory framework for description of M1 transitions, based on the relativistic nuclear energy density functional, provides the insight into spin, orbital, isoscalar and isovector M1 transition strengths in magic and open shell nuclei. Since in open shell nuclei pairing correlations are essential to reproduce the M1 transition strength distributions, this mode could be used as an additional constraint for the nuclear pairing interactions. The evolution of M1 transition properties in ${ }^{100-140} \mathrm{Sn}$ isotopes demonstrate interesting interplay between single and double-peak structures, determined by the evolution of single-particle states, their occupations governed by the pairing correlations, and involvement of various two-quasiparticle configurations. Because M1 excitations originate from transitions between spin-orbit partner states, their properties also represent useful constraint for the spin-orbit interaction channel. From the comparison with the inelastic proton scattering data for ${ }^{112-124} \mathrm{Sn}$, it is shown that quenching in the $g$ factors needed to reproduce the experimental data amounts $g_{\text {eff }} / g_{\text {free }}=0.80$ 0.93 , that is larger value than known from previous studies, $g_{\text {eff }} / g_{\text {free }} \approx 0.6-0.75$. Since some measured B(M1) strength may be missing around the neutron threshold, further investigations are required to determine the quenching factor accurately.

\section{Acknowledgments}

This work is supported by the "QuantiXLie Centre of Excellence", a project co-financed by the Croatian Government and European Union through the European Regional Development Fund, the Competitiveness and Cohesion Operational Programme (KK.01.1.1.01). 


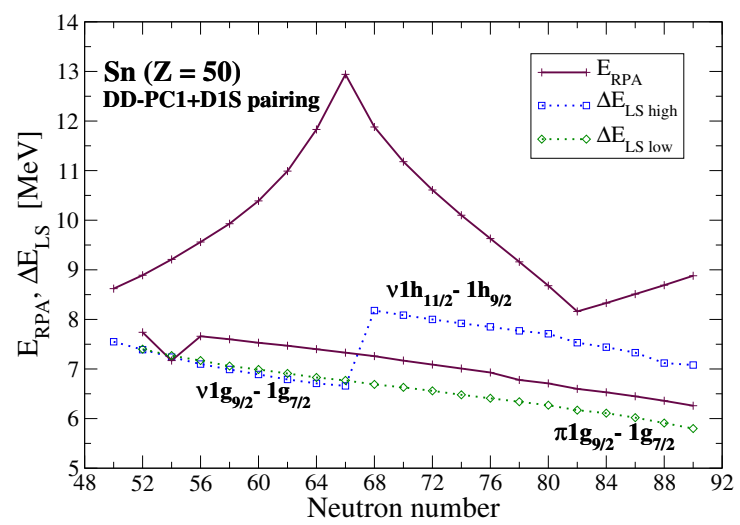

Figure 3. The M1-excitation energies in $\mathrm{Sn}$ isotopes and the corresponding SO-splitting energies $\Delta E_{L S}((\mathrm{nlj})$ quantum numbers are denoted for the SO-partner levels). $\Delta E_{L S_{\text {low }}}$ and $\Delta E_{L S_{\text {high }}}$ represent the SO splitting contributions at lower and higher RPA energies, respectively.

\section{References}

[1] A. Richter, Progress in Particle and Nuclear Physics 34, 261 (1995)

[2] K. Heyde, P. von Neumann-Cosel, A. Richter, Rev. Mod. Phys. 82, 2365 (2010)

[3] N. Pietralla, P. von Brentano, A. Lisetskiy, Progress in Particle and Nuclear Physics 60, 225 (2008)

[4] V.O. Nesterenko, J. Kvasil, P. Vesely, W. Kleinig, P.G. Reinhard, V.Y. Ponomarev, Journal of Physics G: Nuclear and Particle Physics 37, 064034 (2010)

[5] D.P. Arteaga, P. Ring, Phys. Rev. C 77, 034317 (2008)

[6] S. Goriely, S. Hilaire, S. Péru, M. Martini, I. Deloncle, F. Lechaftois, Phys. Rev. C 94, 044306 (2016)

[7] T. Otsuka, T. Suzuki, M. Honma, Y. Utsuno, N. Tsunoda, K. Tsukiyama, M. Hjorth-Jensen, Phys. Rev. Lett. 104, 012501 (2010)

[8] P. Vesely, J. Kvasil, V.O. Nesterenko, W. Kleinig, P.G. Reinhard, V.Y. Ponomarev, Phys. Rev. C 80, 031302 (2009)

[9] V. Tselyaev, N. Lyutorovich, J. Speth, P.G. Reinhard, D. Smirnov, Phys. Rev. C 99, 064329 (2019)

[10] M.B. Chadwick, et. al., Nuclear Data Sheets 112, 2887 (2011)

[11] J.D. Vergados, H. Ejiri, F. Šimkovic, Reports on Progress in Physics 75, 106301 (2012)

[12] K. Langanke, G. Martínez-Pinedo, P. von NeumannCosel, A. Richter, Phys. Rev. Lett. 93, 202501 (2004), and references therein.

[13] H.P. Loens, K. Langanke, G. Martinez-Pinedo, K. Sieja, The European Physical Journal A 48, 34 (2012)

[14] K. Heyde, P. von Neumann-Cosel, A. Richter, Rev. Mod. Phys. 82, 2365 (2010)

[15] J.P. Ebran, E. Khan, D. Peña Arteaga, D. Vretenar, Phys. Rev. C 83, 064323 (2011)

[16] A. Tamii, T. Adachi, J. Carter, M. Dozono, H. Fujita, Y. Fujita, K. Hatanaka, H. Hashimoto, T. Kaneda,
M. Itoh et al., Nuclear Physics A 788, 53 (2007), proceedings of the 2nd International Conference on Collective Motion in Nuclei under Extreme Conditions

[17] Y. Fujita, B. Rubio, W. Gelletly, Prog. Part. Nucl. Phys. 66, 549 (2011)

[18] J. Birkhan, H. Matsubara, P. von Neumann-Cosel, N. Pietralla, V.Y. Ponomarev, A. Richter, A. Tamii, J. Wambach, Phys. Rev. C 93, 041302 (2016)

[19] R.M. Laszewski, R.J. Holt, H.E. Jackson, Phys. Rev. Lett. 38, 813 (1977)

[20] J. Speth, P.G. Reinhard, V. Tselyaev, N. Lyutorovich, arXiv:2001.07236 (2020)

[21] G. Kružić, T. Oishi, D. Vale, N. Paar, Phys. Rev. C 102, 044315 (2020)

[22] T. Oishi, G. Kružić, N. Paar, Journal of Physics G: Nuclear and Particle Physics 47, 115106 (2020)

[23] G. Kružić, T. Oishi, N. Paar, Phys. Rev. C 103, 054306 (2021)

[24] T. Oishi, G. Kružić, N. Paar, Eur. Phys. J. A 57, 180 (2021)

[25] T. Oishi, N. Paar, Phys. Rev. C 100, 024308 (2019)

[26] S. Bassauer, P. von Neumann-Cosel, P.G. Reinhard, A. Tamii, S. Adachi, C.A. Bertulani, P.Y. Chan, A. D’Alessio, H. Fujioka, H. Fujita et al., Phys. Rev. C 102, 034327 (2020)

[27] R. Alarcon, R.M. Laszewski, D.S. Dale, Phys. Rev. C 40, R1097 (1989)

[28] K. Govaert, F. Bauwens, J. Bryssinck, D. De Frenne, E. Jacobs, W. Mondelaers, L. Govor, V.Y. Ponomarev, Phys. Rev. C 57, 2229 (1998)

[29] P. Vesely, J. Kvasil, V.O. Nesterenko, W. Kleinig, P.G. Reinhard, V.Y. Ponomarev, Phys. Rev. C 80, 031302(R) (2009)

[30] V.O. Nesterenko, J. Kvasil, P. Vesely, W. Kleinig, P.G. Reinhard, V.Y. Ponomarev, Journal of Physics G: Nuclear and Particle Physics 37, 064034 (2010)

[31] M. Ichimura, H. Sakai, T. Wakasa, Progress in Particle and Nuclear Physics 56, 446 (2006) 